\title{
Large area single-mode parity-time-symmetric laser amplifiers
}

\author{
Mohammad-Ali Miri,* Patrik LiKamWa, and Demetrios N. Christodoulides \\ CREOL, College of Optics and Photonics, University of Central Florida, Orlando, Florida 32816-2700, USA \\ *Corresponding author: miri@knights.ucf.edu \\ Received October 26, 2011; revised January 9, 2012; accepted January 15, 2012; \\ posted January 17, 2012 (Doc. ID 157003); published February 17, 2012
}

\begin{abstract}
By exploiting recent developments associated with parity-time (PT) symmetry in optics, we here propose a new avenue in realizing single-mode large area laser amplifiers. This can be accomplished by utilizing the abrupt symmetry breaking transition that allows the fundamental mode to experience gain while keeping all the higher order modes neutral. Such PT-symmetric structures can be realized by judiciously coupling two multimode waveguides, one exhibiting gain while the other exhibits an equal amount of loss. Pertinent examples are provided for both semiconductor and fiber laser amplifiers. (ㄷ) 2012 Optical Society of America

OCIS codes: $140.3570,140.4480,130.2790$.
\end{abstract}

High-power laser amplifiers nowadays play a crucial role in optics. Their applications range from cutting and welding to optically pumping other laser systems. In all occasions, extreme care is taken to avoid unwanted side effects arising from the delivery of such high power levels. These include, among others, nonlinear processes such as stimulated Raman and Brillouin scattering effects. Scaling up the cross section of the gain medium provides a natural way to achieve this goal. Not only does it lead to higher output powers, but it also provides a solution in reducing the impact of nonlinear effects. Unfortunately however, such an increase in size comes at a price: it makes the structure multimoded. This in turn has a detrimental effect on the output beam quality and the temporal stability of the laser itself.

In order to force these large area optical amplifiers to only lase in their fundamental mode, several strategies have been suggested. For broad area semiconductor laser amplifiers, the majority of these methods relies on spatial filtering. Modal reflectors [1], external cavities $[2,3]$, and distributed feedback gratings [4] have been used as a means to increase the loss associated with higher order modes. Another approach is based on using tapers to gradually increase the width of the device while exciting only the fundamental mode [5]. For fiber laser amplifiers, on the other hand, several other approaches have also been proposed to address this problem. One way is to use large area endlessly single-mode photonic crystal fibers [6] or leakage channels structures [7]. Other schemes utilize the distributed loss offered by coiled fibers to filter out higher order modes [8]. Selective excitation of the fundamental mode using ultrashort pulses was demonstrated in multimode fibers [9], while spatial doping has been used to achieve gain filtering among different modes [10]. Gain guiding with index antiguiding provides yet another technique for single-mode large area lasers [11]. Given that none of the aforementioned methods can single-handedly address all the underlying problems in this area, of interest will be to explore alternative routes to achieve this goal for both one- and two-dimensional structures in different geometries.
In this Letter, we propose a novel avenue in order to encourage single-mode operation of large area optical amplifiers. This is done by exploiting recently developed notions in parity-time (PT) symmetric optics. As we will see, what distinguishes this new class of systems from the previously mentioned schemes is that only the fundamental mode experiences gain while all the higher order modes undergo oscillations and hence remain neutral.

The concept of PT symmetry first emerged within the context of mathematical physics. In this regard, it was recognized that a wide class of non-Hermitian Hamiltonians can exhibit entirely real spectra as long as they commute with the PT operator [12]. Lately, these notions have been successfully extended and observed in optics by utilizing the isomorphism between the respective evolution equations [13-17].

To appreciate some of the ramifications of this symmetry in the optical domain, let us consider for simplicity a structure composed of two identical multimode waveguides coupled to each other (Fig. 1). In this case, PT symmetry around the central axis demands that one of the waveguides exhibits gain while the other exhibits an equal amount of loss [15]. By considering only the coupling effects between identical modes, the evolution of the modal amplitudes $a_{m}$ and $b_{m}$ of the $m^{\prime}$ th modes in these two guides is described through the coupled mode equations:

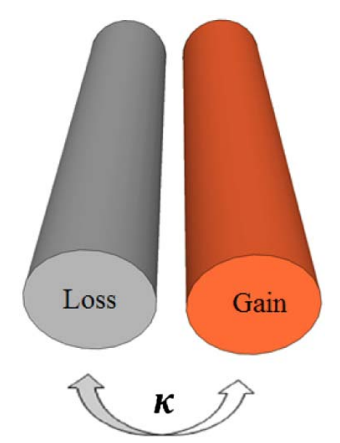

Fig. 1. (Color online) Two PT-symmetric coupled dielectric multimode waveguides. 


$$
\begin{gathered}
\frac{d a_{m}}{d z}=i \beta_{m} a_{m}+i \kappa_{m} b_{m}+g_{m} a_{m}, \\
\frac{d b_{m}}{d z}=i \beta_{m} b_{m}+i \kappa_{m} a_{m}-g_{m} b_{m},
\end{gathered}
$$

where $\beta_{m}$ is their respective propagation constant, $\kappa_{m}$ is coupling coefficient among these modes, and $\pm g_{m}$ stands for the modal gain or loss in the $m^{\prime}$ th mode.

The solution of these coupled wave equations, can be obtained through their respective supermodes. For convenience, we introduce the dimensionless quantity $\rho_{m}=\frac{g_{m}}{\kappa_{m}}$. Two regimes are identified. If the system is kept below threshold $\left(\rho_{m}<1\right)$, the two supermodes are

$$
\left(\begin{array}{l}
a_{m} \\
b_{m}
\end{array}\right)=\left(\begin{array}{c}
1 \\
\pm \exp \left( \pm i \theta_{m}\right)
\end{array}\right) \exp \left( \pm i \kappa \cos \left(\theta_{m}\right) z\right) \exp \left(i \beta_{m} z\right)
$$

where $\sin \left(\theta_{m}\right)=\rho_{m}$. Note that in this case none of the modes experiences gain-instead they both remain neutral and therefore oscillate during propagation. If on the other hand, $\rho_{m}>1$, then

$$
\left(\begin{array}{l}
a_{m} \\
b_{m}
\end{array}\right)=\left(\begin{array}{c}
1 \\
i \exp \left( \pm \theta_{m}\right)
\end{array}\right) \exp \left(\mp \kappa \sinh \left(\theta_{m}\right) z\right) \exp \left(i \beta_{m} z\right)
$$

where $\cosh \left(\theta_{m}\right)=\rho_{m}$. In this case, the PT symmetry is "spontaneously broken" and hence one of the two supermodes enjoys amplification while the other decays exponentially with distance. The limit $\rho_{m}=1$ designates this transition point.

To understand how the proposed arrangement works, one has to bear in mind that the coupling coefficient between higher order modes is typically higher than that for lower ones such as the fundamental. Hence, for a given gain level, $\rho_{m}$ is expected to be higher for lower order modes. If the system is appropriately designed, then only the fundamental mode will exhibit a ratio, $\rho_{1}>1$ while the rest are kept below unity. As a result, only the fundamental mode will experience PT-symmetry breaking and thus will be amplified. On the other hand, the rest of the modes will be neutral and therefore remain bounded in amplitude [as in Eq. (2)], exhibiting oscillations.

In what follows, we provide pertinent examples to elucidate this possibility. First we consider a semiconductor amplifier waveguide system consisting of two identical PT-symmetric ridge guides in contact to each other [Fig. 2(a)]. The index in this region is the same throughout while one guide experiences gain and the other an equal amount of loss. Each waveguide is taken here to be $30 \mu \mathrm{m}$ thick, and the operating wavelength is assumed to be $1 \mu \mathrm{m}$. The substrate has a refractive index of 3.5, while the core has a relatively high index contrast of 0.003 compared to the substrate so as to discourage any beam filamentation effects arising from spatial hole burning. A bulk gain/loss of $\pm 5.906 \mathrm{~cm}^{-1}$ is assumed in these two regions of this waveguide. This structure is analyzed using finite difference schemes. Figure 2(b) depicts the contrast in refractive index profile as well as the imaginary part of the refractive index. Table 1 provides the effective index of the first six TE modes, while
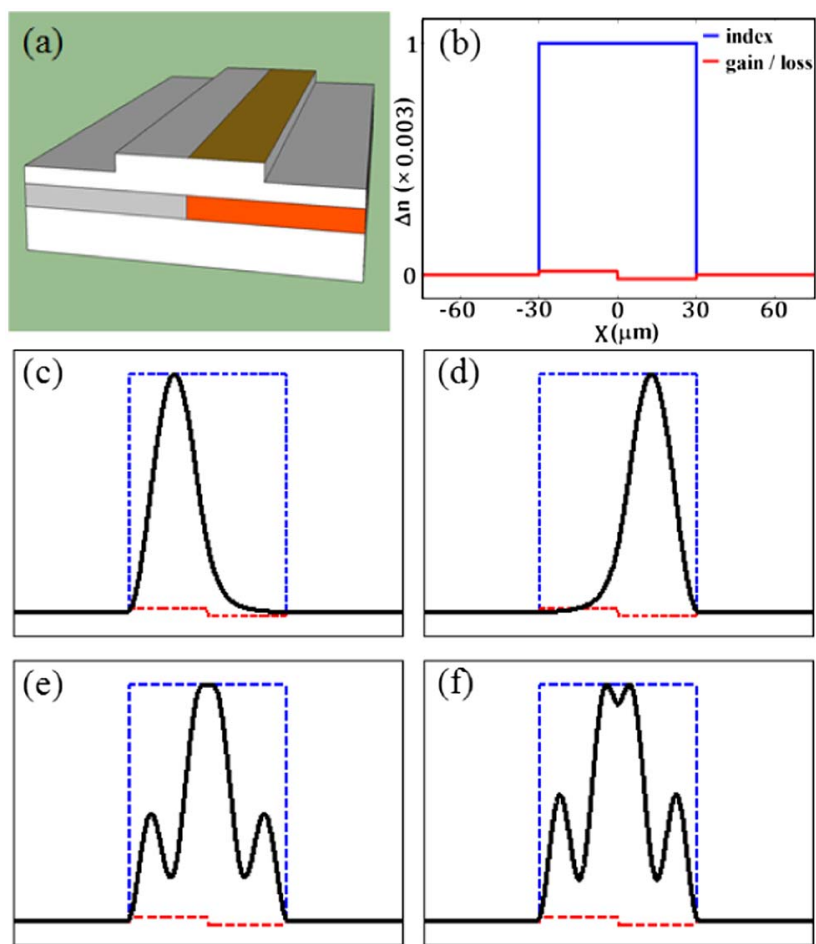

Fig. 2. (Color online) PT-symmetric semiconductor laser amplifier: (a) schematic of the laser, (b) real and imaginary index (gain/loss) profiles, and (c)-(f) field intensity profile of the first TE modes.

the intensity profile of the first four is depicted in Figs. 2(c)-(f). Evidently, only the first pair of supermodes (corresponding to the fundamental $\mathrm{TE}_{0}$ in each region) is in the broken phase regime, while the rest of the modes lie below the threshold and hence they are neutral. Figure 2 illustrates the main difference between broken phase modes and ordinary modes in this PT-symmetric structure. For modes kept below the threshold, the optical intensity is symmetric, while for those with broken symmetry, it is asymmetric [Figs. 2(c), (d)]. Note that one of these latter modes mostly lies in the gain region and is hence amplified, while the other one occupies the loss region and is attenuated.

We also analyze a two-dimensional PT fiber based system. In such arrangements, higher order modes may not necessarily have the highest coupling. In fact, the coupling in this case depends on the nature of the mode itself [18]. As an example, we consider two circular cores, each having a diameter of $60 \mu \mathrm{m}$ in contact with each other. Such double-core arrangements may be feasible by appropriately structuring the fiber preform [19]. The refractive index of the core and cladding regions is assumed to

Table 1. Complex Effective Indices of the First TE Modes of a Large Area Semiconductor Laser

\begin{tabular}{lcc}
\hline Mode Number & Complex Effective Index & Gain/Loss $\left(\mathrm{cm}^{-1}\right)$ \\
\hline $\mathrm{TE}_{0}$ & $3.502973+i 0.00003976$ & -5 \\
$\mathrm{TE}_{0}$ & $3.502973-i 0.00003976$ & +5 \\
$\mathrm{TE}_{1}$ & 3.502891 & 0 \\
$\mathrm{TE}_{1}$ & 3.502877 & 0 \\
$\mathrm{TE}_{2}$ & 3.502765 & 0 \\
$\mathrm{TE}_{2}$ & 3.502674 & 0 \\
\hline
\end{tabular}




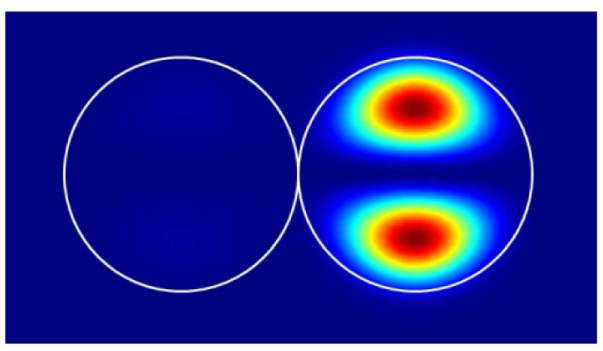

Fig. 3. (Color online) Intensity profile of the $\mathrm{LP}_{11}$ modes experiencing symmetry breaking.

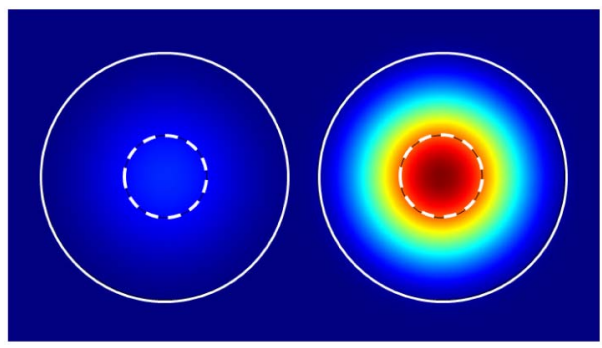

Fig. 4. (Color online) Intensity profile of the broken $\mathrm{LP}_{01}$ mode in a PT-symmetric multimode coupled system. Gain/loss is provided within the dashed circular regions.

be 1.535 and 1.534 , respectively, corresponding to a NA of 0.055 . A differential gain/loss of $\pm 0.5 \mathrm{~cm}^{-1}$ is assumed, which is typical of phosphate glass laser amplifiers [20]. The operating wavelength is $1.54 \mu \mathrm{m}$.

According to finite element simulations, the fundamental $\mathrm{LP}_{01}$ is not the first mode to experience symmetry breaking. Instead, two degenerate pairs (one for each polarization) of the $\mathrm{LP}_{11}$ supermodes are the first to break the PT symmetry in this example, experiencing a gain/loss of $\pm 0.4541 \mathrm{~cm}^{-1}$. This result can be explained through Fig. 3, which shows the intensity profile for the $x$-polarized $\mathrm{L} \overline{\mathrm{P}}_{11}$ mode of this structure. As this figure indicates, this specific set of $\mathrm{LP}_{11}$ modes has very small overlap, and thus their coupling coefficient is lower than that of the fundamental $\mathrm{LP}_{01}$. As a result, they are the first to break the PT symmetry.

To overcome this problem, we confine the gain/loss process in two cocentric cylindrical regions of $20 \mu \mathrm{m}$ diameter, as shown in Fig. 4. In this case, the two cores are also further separated by $6.8 \mu \mathrm{m}$ to decrease the coupling constants. Figure 4 . shows the intensity profile of the first few $x$-polarize $\bar{d}$ modes of this new structure. In this case, the fundamental $\mathrm{LP}_{01}$ mode is the first to break the symmetry, and its modal gain/loss is approximately $\pm 0.1 \mathrm{~cm}^{-1}$. Thus, this coupled multimode PT-symmetric structure is expected to lase only in the fundamental mode (of the gain region), while all higher order modes will remain neutral.

Finally it is important to note that many of these features associated with PT symmetry can actually persist in spite of imperfections and perturbations, such as, for example, bending, thermal, and saturation effects that may spoil the assumed symmetry. In principle, appreciable losses can be introduced to the system by scattering regions so as to avoid any unnecessary thermal effects because of absorption. However, on many occasions, this perfect symmetry may not be absolutely essential in exploiting these effects. For example, let us consider a perturbed PT system where the $m^{\prime}$ th mode in the first waveguide experience a modal gain of $+g_{m}^{(1)}$ while this same mode in the second waveguide experiences a loss of $-g_{m}^{(2)}$. In this case, if we define a common gain/loss factor of $\pm g_{m}= \pm\left(g_{m}^{(1)}+g_{m}^{(2)}\right) / 2$, it is straightforward to show that Eqs. (2) and (3) still hold under these same conditions, provided that a net gain (or loss depending on the sign) of $\left(g_{m}^{(1)}-g_{m}^{(2)}\right) / 2$ is added to all modes. Thus, some of the higher order modes may no longer be neutral. Yet, even in the presence of such imperfections, single-mode operation can be restored by appropriately shifting the overall zero gain/loss line of the structure. Essentially, perturbations in the spectrum tend to increase linearly with the strength of such asymmetries.

In conclusion, we proposed a new method to achieve single-mode operation of broad area multimode laser amplifiers. This scheme is based on the notion of PTsymmetry breaking and is applicable to both one- and two-dimensional configurations.

\section{References}

1. M. Szymanski, J. M. Kubica, P. Szczepanski, and B. Mroziewicz, J. Phys. D 30, 1181 (1997).

2. L. Goldberg and J. F. Weller, Electron. Lett. 25, 112 (1989).

3. S. Wolff, D. Messerschmidt, and H. Fouckhardt, Opt. Express 5, 32 (1999).

4. R. J. Lang, K. Dzurko, A. A. Hardy, S. Demarrs, A. Schoenfelder, and D. F. Welch, IEEE J. Quantum Electron. 34, 2196 (1998).

5. J. N. Walpole, E. S. Kintzer, S. R. Chinn, C. A. Wang, and L. J. Missaggia, Appl. Phys. Lett. 61, 740 (1992).

6. J. Limpert, T. Schreiber, S. Nolte, H. Zellmer, T. Tunnermann, R. Iliew, F. Lederer, J. Broeng, G. Vienne, A. Petersson, and C. Jakobsen, Opt. Express 11, 818 (2003).

7. L. Dong, X. Peng, and J. Li, J. Opt. Soc. Am. B 24, 1689 (2007).

8. J. P. Koplow, D. A. V. Kliner, and L. Goldberg, Opt. Lett. 25, 442 (2000).

9. M. E. Fermann, Opt. Lett. 23, 52 (1998).

10. J. R. Marciante, IEEE J. Sel. Top. Quantum Electron. 15, 30 (2009).

11. A. E. Siegman, Y. Chen, V. Sudesh, M. C. Richardson, M. Bass, P. Foy, W. Hawkins, and J. Ballato, Appl. Phys. Lett. 89, 251101 (2006).

12. C. M. Bender and S. Boettcher, Phys. Rev. Lett 80, 5243 (1998).

13. A. Guo, G. J. Salamo, D. Duchesne, R. Morandotti, M. Volatier-Ravat, V. Aimez, G. A. Siviloglou, and D. N. Christodoulides, Phys. Rev. Lett. 103, 093902 (2009).

14. C. E. Ruter, K. G. Makris, R. El-Ganainy, D. N. Christodoulides, M. Segev, and D. Kip, Nat. Phys. 6, 192 (2010).

15. R. El-Ganainy, K. G. Makris, D. N. Christodoulides, and Z. H. Musslimani, Opt. Lett. 32, 2632 (2007).

16. K. G. Makris, R. El-Ganainy, D. N. Christodoulides, and Z. H. Musslimani, Phys. Rev. Lett. 100, 103904 (2008).

17. S. Longhi, Phys. Rev. A 82, 031801(R) (2010).

18. K. Ogawa, Bell Syst. Tech. J. 56, 729 (1977).

19. Y. W. Lee, M. J. F. Digonnet, S. Sinha, K. E. Urbanek, R. L. Byer, and S. Jiang, IEEE J. Sel. Top. Quantum Electron. 15, 93 (2009).

20. S. Jiang, T. Luo, B. C. Hwang, F. Smekatala, K. Seneschal, J. Lucas, and N. Peyghambarian, J. Non-Cryst. Solids 263-264, 364 (2000). 